\title{
Análisis Comparativo de Índices de Calidad del Agua Aplicados al Río Ranchería, La Guajira-Colombia
}

\author{
Jhonny I. Pérez ${ }^{(1)}$, Andrea G. Nardini( ${ }^{(2)}$ y Andrés A. Galindo(1) \\ (1) Grupo de investigación GISA, Facultad de ingeniería, Universidad de La Guajira, km 5 vía Maicao, \\ Riohacha-Colombia. (e-mail: jpemon@uniguajira.edu.co; agalindo@uniguajira.edu.co) \\ (2) Fundación CREACUA-Centro Recuperación Ecosistemas Acuáticos, calle 1ํㅗำ 1-109, Riohacha, La \\ Guajira-Colombia. (e-mail: nardiniok@gmail.com)
}

Recibido Ago. 17, 2017; Aceptado Oct. 20, 2017; Versión final Dic. 6, 2017, Publicado Jun. 2018

\begin{abstract}
Resumen
Este artículo compara diversos índices de calidad del agua (ICA) aplicados en 235,3 km del río Ranchería, La Guajira-Colombia. La comparación de los ICA se realizó directamente con los valores numéricos de los índices discretizados en cinco rangos iguales (índices brutos) y con sus calificaciones, es decir los juicios ambientales (también en cinco clases) asociados a esos valores. La coherencia entre parejas de índices se analizó en ambos casos con el coeficiente de correlación de Spearman y la raíz del error cuadrático medio (RMSE). Se obtuvo mayor coincidencia, mejor correlación y menores desviaciones en el caso de los índices brutos. Este hecho evidencia la carencia de la relación entre los valores de los índices y su calificación. Se determina por tanto la necesidad de llevar a cabo un ejercicio de intercalibración para asegurar la coherencia entre los distintos índices.
\end{abstract}

Palabras clave: índices de calidad del agua; análisis comparativo; juicios ambientales; usos del agua

\section{Comparative Analysis of Water Quality Indices Applied to the Ranchería River, La Guajira-Colombia}

\begin{abstract}
This paper compares several water quality indices applied along $235.3 \mathrm{~km}$ of the river Ranchería, La GuajiraColombia. The comparison directly adopts the values of the indices discretized in five equal intervals (raw indices), and, on their scoring, this is the environmental judgments (also in five classes) associated with such indices. The coincidence between couples of indices is analyzed, in both cases, through the correlation coefficient of Spearman and the Root Mean Square Error (RMSE). The results state that raw indices perform higher coincidence, better correlation and lesser deviations than do scorings. This fact stresses the weakness of the relation between the value of the indices and their qualification. It is necessary to carry out an intercalibration exercise in order to consistently gear the different indices.
\end{abstract}




\section{INTRODUCCIÓN}

Los recursos hídricos desempeñan un papel vital en diversos sectores de la economía, como la agricultura, la ganadería, actividades industriales, recreación entre otras. La disponibilidad y calidad del agua, ya sea superficial o subterránea, se ha deteriorado debido a factores como el aumento de la población, la industrialización, la urbanización (Tyagi et al., 2013). La calidad del agua es uno de los temas más importantes en la gestión de los recursos hídricos (Sutadian et al., 2016; Medeiros et al., 2017), su clasificación basada en el grado de pureza y contaminación data de 1848 (Medeiros et al., 2017) y se evalúa desde las características físicas, químicas y bilógicas (Rangeti et al., 2015). La gran variedad de factores y parámetros que afectan la calidad del agua hace que su evaluación sea una tarea compleja (Sutadian et al., 2016). Nace entonces la necesidad de construir índices de calidad del agua (ICAs) como una técnica valiosa para describir su estado general en un solo término de fácil comunicación al público y a los responsables de la toma de decisiones (Tyagi et al., 2013; Sutadian et al., 2016). En 1908, se utilizó uno de los primeros ICA, denominado índice sapróbico, para estimar el nivel de materia orgánica fácilmente degradable en agua superficial (Medeiros et al., 2017) y desde entonces se han desarrollado muchos índices (Sutadian et al., 2016). Los ICAs se han vuelto una herramienta muy útil en los programas de vigilancia, control y administración del recurso hídrico. Colombia los ha incluido como exigencias en el plan de ordenamiento del recurso hídrico $(\mathrm{PORH})$ de cualquier río, como es el caso del río Ranchería (La Guajira-Colombia) a cargo de la Corporación Autónoma Regional de La Guajira (Corpoguajira, 2012).

En muchos casos, los índices adoptan el nombre de la institución o apellido del autor. El índice original de la National Sanitation Foundation (NSF) propuesto por Brown et al., (1970) tiene un núcleo aditivo (NSFa), este puede originar eclipsamiento cuando el índice global tiene un valor satisfactorio aunque un subíndice no lo sea, razón por la cual Brown et al., (1973) propuso un núcleo multiplicativo (NSFm) lo que lo hace más sensible a valores extremos en los subíndices de calidad (Rangeti et al., 2015; Sutadian et al., 2016). En el NSF los subíndices son funciones que transforman el valor de la variable o parámetro en un sub-juicio de calidad. El índice de calidad del agua del río CAUCA llamado ICAUCA (CVC, 2004), el de la Compañía Ambiental del Estado de São Paulo en Brasil (CETESB, 2006), y Dinius (Dinius, 1987) adoptan la fórmula del NSFm. El ICAUCA toma las funciones de valor de los índices: NSF, Dinius y del índice de contaminación por sólidos suspendidos, el cual ha sido usado por Ocampo-Duque et al., (2013). La CETESB (2006) adoptó las mismas ecuaciones del NSF con pequeñas sustituciones, recientemente usado por Medeiros et al., (2017), mientras que Dinius tiene sus propias funciones definidas con un panel de expertos norteamericanos.

En la mayoría de los casos, los índices son diseñados para ríos con calidad y condiciones diferentes. El índice de grado de Cumplimiento (IGC) y el ICAUCA fueron diseñados en Colombia, aunque este último es una adaptación de varios índices. Los ICAs que más se alejan de nuestro contexto climático y ambiental son: NSF y Dinius, pero se usan en muchos países. El IGC fue desarrollado por Ávila y Zuñiga en el 2007 en el marco de un proyecto de Cooperación (Corpoguajira y Ricerca e Cooperazione, 2007) tiene un núcleo aditivo, cuyos subíndices, en este caso, simplemente cuentan la frecuencia con que los parámetros cumplen los estándares respectivos establecidos. La ventaja del IGC está en su simpleza, posibilidad de tratar casos con datos faltantes y fácil interpretación para adaptar a cualquier país o legislación. Un caso similar al IGAC es el ICA del Consejo Canadiense del Ministerio del Ambiente (CCME) diseñado en función de los estándares de calidad de ese país (Hurley et al., 2012).

No obstante la comprobada utilidad, los índices no son exentos de debilidades. Nardini (2003) identificó claramente las debilidades principales de los índices clásicos como el NSF, las cuales se resumen parcialmente a continuación: i) Falta de claridad en relación a que satisfacción intentan medir, porque el índice puede representar bien a un interés -ejemplo la idoneidad del suministro para consumo humano- pero no a los demás: cada uso tienen exigencias diferentes y el índice no las distingue porque no está construido explícitamente para cada una. ii) En general, los índices no se basan en atributos naturales y, por tanto, incorporan implícitamente un modelo causa-efecto entre la calidad objetiva y el efecto que se quiere medir; típicamente contempla la superación de un umbral de concentración de algún compuesto tóxico sin explicitar por tanto la relación entre dicha concentración y el efecto sobre la salud medible a través de la morbilidad y/o mortalidad. iii) Muy a menudo, los índices no respetan la coherencia interna, es decir situaciones físicamente diversas que arrojan el mismo valor numérico del índice no resultan indiferentes frente al tipo de satisfacción que se pretende medir.

Los ICAs utilizados en este estudio se seleccionaron en función de la popularidad de su uso nacional e internacional, la inclusión de variables que afecten la calidad del cuerpo de agua considerado (río Ranchería) y la aptitud para aplicarse a ríos de zonas tropicales. El índice simplificado de calidad de agua (ISCA) de España que evalúa la calidad del agua para varios usos no fue considerado por no incluir los coliformes fecales (CF) que es un parámetro que afecta considerablemente al río Ranchería y a la gran mayoría de los 
ríos en el mundo, lo que hace a estas aguas no aptas para actividades de contacto directo y riego de verduras y frutas que se consumen en crudo o que se desarrollan a ras del suelo (Rivera et al., 2009).

Consientes de estas limitaciones, decidimos evaluar de forma comparativa la coherencia de los ICAs más utilizados que se diferencian por parámetros (variables) considerados, rangos de clases y juicios de calidad, lo cual complica su comparación y entendimiento cuando se aplican a una misma fuente de agua. Por tanto, el objetivo de esta publicación es verificar qué tan consistentes son los ICAs utilizados, apoyar su entendimiento para optimizar su uso y eventualmente detectar la necesidad de diseñarlos de acuerdo al contexto. Además se aporta conocimiento para la planificación del recurso hídrico y facilitar el proceso de optimización de los usos de la fuente hídrica considerada. El caso de estudio es el río Ranchería (La GuajiraColombia) tomando datos de campaña realizada en el mes de febrero de 2012 (época seca). Los resultados de los ICAs fueron comparados con los rangos brutos y clasificación para determinar las coincidencias en cada índice. La información histórica de calidad del agua fue utilizada como referente del estado y además para definir las estaciones de monitoreo y diseñar la campaña de muestreo ulterior realizada expresamente en febrero de 2012. Es con estos datos que se evaluaron los ICAs analizados.

\section{MATERIALES Y MÉTODOS}

La metodología se presenta en tres secciones: la primera describe el área de estudio, la segunda definen las estaciones, el muestreo y el tratamiento de las muestras, y por último se presentan los índices seleccionados y el proceso seguido para su comparación.

\section{Área de estudio}

El rio Ranchería (La Guajira-Colombia) es la principal fuente hídrica superficial del departamento de La Guajira con una longitud total de $284 \mathrm{~km}$, nace en la Sierra Nevada de Santa Marta (Corpoguajira, 2012). La evaluación de los ICAs se realizó desde aguas abajo de la represa EI Cercado a $10^{\circ} 54^{\prime} 30,06^{\prime \prime}$ latitud norte y $73^{\circ} 00^{\prime}$ $36,94^{\prime \prime}$ longitud oeste hasta su desembocadura en el mar Caribe a N: $11^{\circ} 33^{\prime} 16,1^{\prime \prime}$ latitud norte y $72^{\circ} 54^{\prime} 10,5^{\prime \prime}$ longitud oeste, lo que representa $235,3 \mathrm{~km}$ (Figura 1).

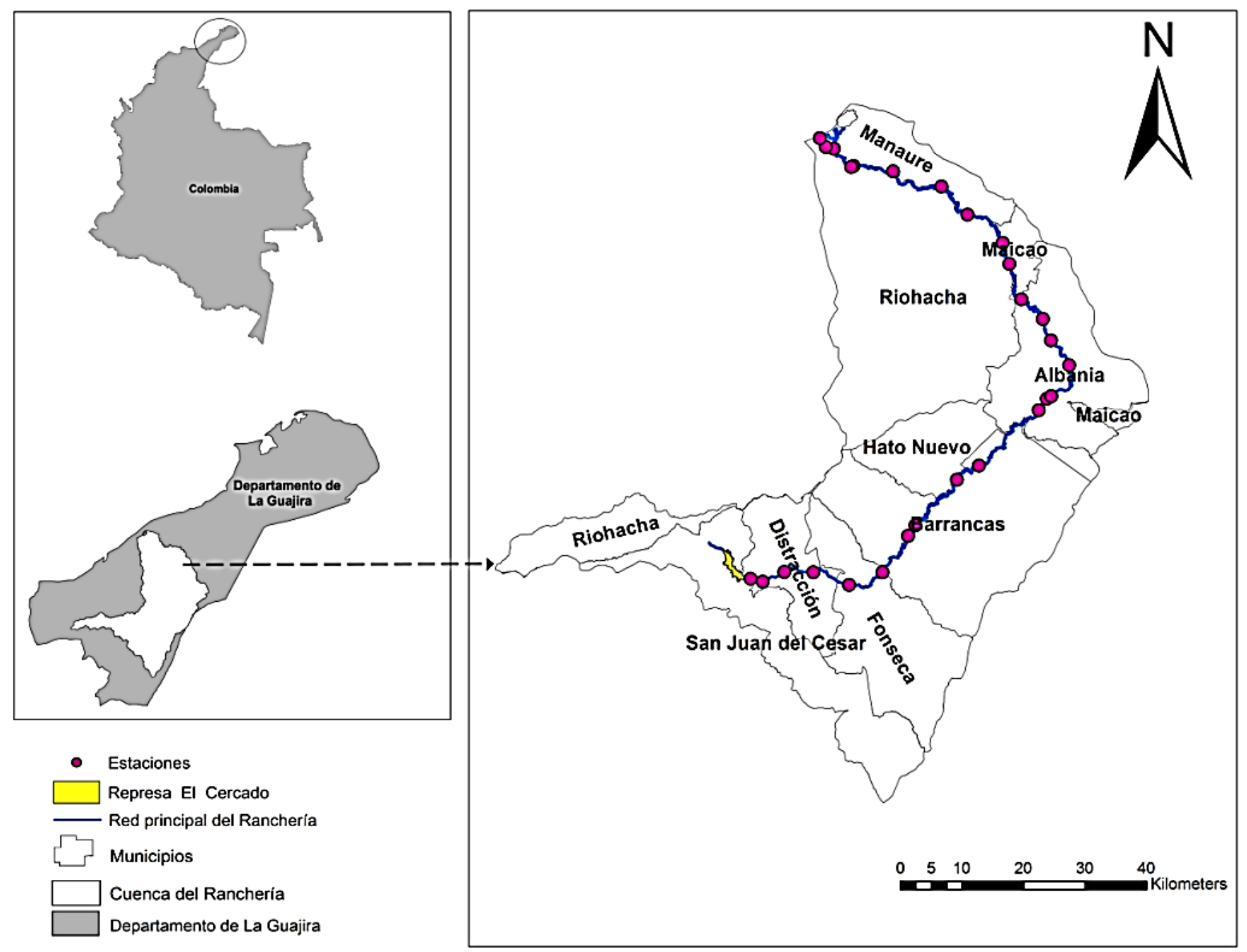

Fig. 1: Ubicación de las estaciones de monitoreo en el río Ranchería, La Guajira- Colombia 


\section{Estaciones de monitoreo}

Antes de la selección de las estaciones de monitoreo, se recopiló y analizó la información histórica disponible en las siguientes entidades: Corpoguajira, Instituto de Investigaciones Marinas y Costeras-INVEMAR, Universidad de La Guajira, Instituto Colombiano de Desarrollo Rural-INCODER y Carbones del Cerrejón Limited-Cerrejón). Conociendo los contaminantes principales y la variabilidad de la calidad del agua en las estaciones históricas, se seleccionaron 27 estaciones definitivas (Figura 1) considerando cuatro criterios: mantener las existentes cuando tuviesen datos históricos significativos, cubrir toda la longitud del tramo en estudio, mantenerse alejados de cualquier confluencia para asegurar que haya ocurrido la mezcla completa para garantizar la representatividad del dato, y contar con al menos una estación por cada tramo geomorfológico. La clasificación por tramos se hizo de acuerdo al enfoque simplificado de estilo de ríos (Brierley et al., 2011). El muestreo fue realizado en época seca (febrero de 2012). In-situ se determinaron: oxígeno disuelto, $\mathrm{pH}$, turbidez, conductividad eléctrica, temperatura ambiente, temperatura del agua; los demás parámetros necesario para el cálculo de los ICAs (DBO, DQO, nitrógeno total, nitrito, nitrato, amonio, fósforo total, fosfato, sólidos totales, sólidos suspendidos, dureza total, alcalinidad, cloruros, sulfato, magnesio, manganeso, calcio, coliformes fecales y totales) fueron realizados en el laboratorio de calidad de agua de la universidad del Magdalena. En todos los casos se procedió de acuerdo con las técnicas analíticas contempladas en el Método Estándar (APHA et al., 2005).

\section{Cálculo de los índices de calidad aplicados al río Ranchería}

Para realizar los cálculos se siguió el procedimiento establecido en cada una de las referencias citadas en la Tabla 1. Para el caso del NSF se usó la forma aditiva (NSFa) y la multiplicativa (NSFm). Para calcular la calidad de cada parámetro en el NSF y CETESB se usó el software ICATest v1.0, versión libre. Los demás cálculos se realizaron en una hoja electrónica específicamente desarrollada en el programa Microsoft office Excel 2010 ®. Los ICAs Dinius e IGC contemplan varios usos de agua, los restantes solo evalúan la calidad del agua para uso y consumo humano previo tratamiento

Tabla 1: Índices de calidad del agua aplicados en la comparación de la medición en el rio Ranchería (La Guajira-Colombia), febrero de 2012.

\begin{tabular}{|c|c|c|c|c|}
\hline Índice & Autor, País de origen & Fundamento & Aplicación & Referencia \\
\hline NSF & $\begin{array}{l}\text { National Sanitation } \\
\text { Foundation, } 1970 \\
\text { (EUA) }\end{array}$ & $\begin{array}{l}\text { Basado en la estructura del } \\
\text { índice de Horton y en el método } \\
\text { Delphi para definir los } \\
\text { parámetros, pesos ponderados, } \\
\text { subíndices li y su clasificación a } \\
\text { ser empleados en el cálculo } \\
\text { (panel de expertos). }\end{array}$ & $\begin{array}{l}\text { Considera el uso para } \\
\text { consumo humano. } \\
\text { Desarrollado para ríos de } \\
\text { EUAy se ha popularizado en } \\
\text { muchos países. }\end{array}$ & $\begin{array}{l}\text { Sutadian } \\
\text { et al., } 2016\end{array}$ \\
\hline ICAUCA & $\begin{array}{l}\text { Corporación del Valle } \\
\text { del Cauca y } \\
\text { Univ.rsidad del Valle, } \\
2004 \text { (Colombia). }\end{array}$ & $\begin{array}{l}\text { Partieron de los ICAs: NSF, } \\
\text { CETESB Dinius entre otros } \\
\text { para crear un nuevo índice } \\
\text { ICAUCA. }\end{array}$ & $\begin{array}{l}\text { Uso para consumo humano. } \\
\text { Es adaptado a las } \\
\text { condiciones ambientales del } \\
\text { río Cauca, Colombia. }\end{array}$ & $\begin{array}{l}\text { CVC, 2004; } \\
\text { Ocampo- } \\
\text { Duque et } \\
\text { al., } 2013\end{array}$ \\
\hline CETESB & $\begin{array}{lr}\text { Companhia } & \text { de } \\
\text { Tecnologia } & \text { de } \\
\text { Saneamento } & \\
\begin{array}{l}\text { Ambiental, } \\
\text { (Brasil) }\end{array} & 2002 \\
\end{array}$ & $\begin{array}{l}\text { Es el mismo NSFm, solo } \\
\text { sustituyeron los parámetros } \\
\text { nitratos y fosfatos por nitrógeno } \\
\text { total y fósforo total } \\
\text { respectivamente. }\end{array}$ & $\begin{array}{l}\text { Uso para consumo humano } \\
\text { ajustado a las condiciones } \\
\text { específicas de los ríos del } \\
\text { Estado de Sao Paulo. }\end{array}$ & $\begin{array}{l}\text { CETESB, } \\
2006 ; \\
\text { Medeiros et } \\
\text { al. } 2017\end{array}$ \\
\hline Dinius & Dinius, 1987- (EUA) & $\begin{array}{l}\text { El mismo fundamento y } \\
\text { ecuación del NFSm, pero } \\
\text { incluyó nuevos parámetros, } \\
\text { rangos y recomendaciones para } \\
\text { cada uno de acuerdo al uso del } \\
\text { agua. Se conformó un grupo de } \\
\text { siete expertos norteamericanos. }\end{array}$ & $\begin{array}{l}\text { Considera cinco usos del } \\
\text { recurso: consumo humano, } \\
\text { agrícola, recreativo, pesca y } \\
\text { vida acuática, industrial. Se } \\
\text { basó en la calidad del agua } \\
\text { para condiciones y ríos de } \\
\text { EUA. }\end{array}$ & $\begin{array}{l}\text { Dinius, } \\
1987 ; \\
\text { Sutadian et } \\
\text { al., } 2016\end{array}$ \\
\hline $\begin{array}{l}\text { IGC, } \\
\text { Índice } \\
\text { Grado de } \\
\text { Cumplimi } \\
\text { ento }\end{array}$ & $\begin{array}{l}\text { Avila y Zúñiga, } 2007 \\
\text { (Colombia) }\end{array}$ & $\begin{array}{l}\text { Es una sumatoria ponderada de } \\
\text { los sub-índices li; cada uno de } \\
\text { ellos cuenta la frecuencia de } \\
\text { cumplimiento, en los distintos } \\
\text { muestreos, del respectivo } \\
\text { estándar de calidad fijado por la } \\
\text { norma Colombiana para un } \\
\text { dado uso. }\end{array}$ & 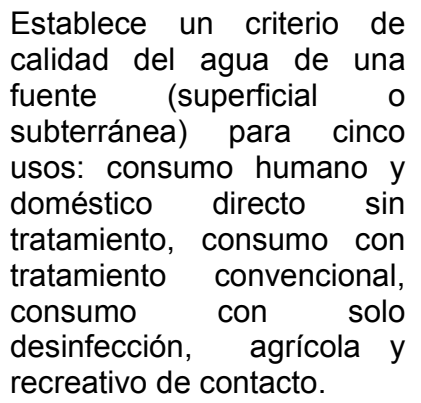 & $\begin{array}{l}\text { Corpoguajir } \\
\text { a y Ricerca } \\
\text { e } \\
\text { Cooperazio } \\
\text { ne, } 2007\end{array}$ \\
\hline
\end{tabular}


Dado que el IGC es poco conocido, se presenta la ecuación 1, usada para su cálculo. Originalmente, fue aplicado en función de los estándares de calidad fijados por la norma colombiana, como es el Decreto 1594 de 1984 (Ministerio de Agricultura, 1984) tomando como caso de estudio el río Tomarrazón Camarones (La Guajira-Colombia).

$$
\mathrm{IGC}^{\mathrm{U}}=\sum_{1}^{\mathrm{n}}\left[\mathrm{w}_{\mathrm{i}}^{\mathrm{U}} * \mathrm{IGC}_{\mathrm{i}}^{\mathrm{U}}\right]
$$

En esta ecuación, $\mathrm{IGC}^{\mathrm{U}}$ es el índice de grado de cumplimiento para el uso del agua $U, \mathrm{w}_{\mathrm{i}}^{\mathrm{U}}$ es el peso asignado al parámetro i de acuerdo al uso $\mathrm{U}, \mathrm{IGC}_{\mathrm{i}}^{\mathrm{U}}$ es el índice de grado de cumplimiento para cada parámetro i, vale: 1 , si el dato de la muestra cumple con todos los estándares de calidad establecidos en la normativa colombiana, cero $(0)$ en caso contrario.

Todos los índices considerados son normalizados entre 0 y 1 y en su estado original plantean cinco rangos de clases para determinar la calidad del agua, excepto el de Dinius que tiene seis clases. Para poderlos comparar y determinar coincidencias entre las parejas de índices, se procedió por un lado directamente con los valores de los índices brutos discretizados, todos, en cinco rangos iguales, es decir 20 puntos cada uno; por otro lado, se compararon las calificaciones que esos índices arrojan en una escala común a todos ellos diseñada explícitamente estableciendo cinco clases de calidad así: 1-excelente, 2-buena, 3-aceptable, 4mala, 5-muy mala (Tabla 2). La traducción del valor del índice bruto a calificación es determinada por una función de valor por intervalos constantes definida por umbrales propios de cada índice, determinados por quien lo diseñó. Para el caso de Dinius se unificaron las clases 5 y 6 por tener cierta similitud de significado: en efecto, la clase 5 es contaminación fuerte -que significa dudosa para consumo humano- y la clase 6 es contaminación en exceso -que es inaceptable para este uso-.

Para conocer la coherencia entre los índices brutos (sin calificación) y las calificaciones, se determinó el coeficiente de correlación $(\rho)$ de Spearman por pareja de índices, este tipo de asociación existe desde 1904 y ha sido ampliamente usado en la actualidad (Blum y Holling, 2017), la cual aplica a variables discretas como es la calificación de los ICAs. Luego se ha complementado el análisis con la raíz del error cuadrático medioRMSE (Mentaschi et a., 2017) que permite detectar de manera sintética las diferencias entre parejas de índices considerando los usos de consumo humano $(\mathrm{CH})$, agrícola (Agr) y recreativo (Rec). Para las parejas de índices se utilizan los umbrales originales que van de 0 a 100, a excepción de la clase 5 del índice de Dinius donde se incluye la clase 6 original).

Tabla 2: Clases adoptadas para la calificación por rango de los índices de calidad del agua en el Rio Ranchería (La GuajiraColombia), febrero de 2012.

\begin{tabular}{|c|l|l|c|c|c|c|c|c|c|}
\hline Clase & Color & Definición & $\begin{array}{c}\text { NSF } \\
\mathrm{CH}\end{array}$ & $\begin{array}{c}\text { ICAUCA } \\
\mathrm{CH}\end{array}$ & $\begin{array}{c}\text { CETESB } \\
\mathrm{CH}\end{array}$ & $\begin{array}{c}\text { Dinius: } \\
\mathrm{CH}\end{array}$ & $\begin{array}{c}\text { Dinius: } \\
\text { Agr. }\end{array}$ & $\begin{array}{c}\text { Dinius: } \\
\text { Rec. }\end{array}$ & $\begin{array}{c}\text { IGC: } \mathrm{CH}, \mathrm{Agr} y \\
\text { Rec. }\end{array}$ \\
\hline 1 & Azul & Excelente & $>90$ & $>80$ & $>79$ & $>90$ & $>90$ & $>70$ & $>90$ \\
\hline 2 & Verde & Buena & $71<1 \leq 90$ & $50<1 \leq 80$ & $51<1 \leq 79$ & $80<1 \leq 90$ & $70<1 \leq 90$ & $50<1 \leq 70$ & $75<1 \leq 90$ \\
\hline 3 & Amarillo & Aceptable & $51<1 \leq 70$ & $35<1 \leq 50$ & $36<1 \leq 51$ & $70<1 \leq 80$ & $50<1 \leq 70$ & $40<1 \leq 50$ & $50<1 \leq 75$ \\
\hline 4 & Naranja & Mala & $26<1 \leq 50$ & $20<1 \leq 35$ & $19<1 \leq 36$ & $50<1 \leq 70$ & $30<1 \leq 50$ & $30<1 \leq 40$ & $25<1 \leq 50$ \\
\hline 5 & Rojo & Muy mala & $0<1 \leq 25$ & $0<1 \leq 20$ & $0<1 \leq 19$ & $0<1 \leq 50$ & $0<1 \leq 30$ & $0<1 \leq 30$ & $0<1 \leq 25$ \\
\hline
\end{tabular}

\section{ANÁLISIS Y DISCUSIÓN DE RESULTADOS}

En la Tabla 3 se observan las comparaciones de las coincidencias y no coincidencias encontradas al comparar las parejas de índices en los distintos usos, así: tomando la primera fila, el contenido "0/1", calificación/índice bruto, debajo de la columna de clase/rango identificada con el número 1, significa que entre los dos índices NSFa y NSFm no hubo ninguna coincidencia de clases de calidad (clasificación), mientras hubo una coincidencia al comparar los rangos (índices brutos).

\section{Consumo humano}

Al hacer las comparaciones entre las distintas parejas de índices, se pudo observar que las mayores coincidencias para la clasificación se presentaron entre el ICAUCA con IGC (12 en clase 2 y 11 en clase 3 ) y NSFa con NSFm (1 en clase 2 y 19 en clase 3). Las clases predominantes en los ICAs son la 2-buena y 3aceptable, excepto Dinius que en su mayoría es clase 4, es decir mala en nuestra escala unificada. Interpretando los juicios o criterios de Dinius, la clase 4 corresponde a contaminación leve, por tanto se puede decir que las clases 2 y 3 del NSF, ICAUCA e IGC son similares a la clase 4 de Dinius. De igual forma se han encontrado clases similares en el lago Dakan-Iraq encontraron clase 2 y 4 (Hameed et al., 2010), el río Gangesa-India clase 4 (Sharma et al., 2014). Analizando las coincidencias por rangos brutos, éstas se incrementaron en parejas diferentes a las que coincidieron en las clases. Las parejas con mayor coincidencias 
fueron NSFm con ICAUCA ( 1 en rango 2 y 25 en rango 3), ICAUCA con CETESB ( 1 en rango 2 y 25 en rango 3 ), NSFa con Dinius (25 en rango 2 ) y NSFm con CETESB (25 en rango 3 ).

En la Figura 2 se presenta una comparación entre los índices NSFm-CETESB, se observa que no existe coincidencia en las calificaciones en ninguna de las 27 estaciones a pesar de ser prácticamente el mismo índice (CETESB usó las mismas funciones de valor, solo cambia fosfato por fosforo total y nitrato por nitrógeno total). Sin embargo, al hacer la comparación por rangos de índices brutos se encontraron 25 coincidencias (el tamaño de la burbuja es proporcional a la numerosidad de casos), corroborando la semejanza del valor directamente resultante en el cálculo del índice, a diferencia de la clasificación.

Tabla 3: Coincidencias entre las parejas de índices en las 27 estaciones de monitoreo comparados en el río Ranchería (La Guajira-Colombia), febrero de 2012.

\begin{tabular}{|c|c|c|c|c|c|c|c|c|}
\hline \multirow[b]{2}{*}{ Parejas de índices } & \multirow[b]{2}{*}{ uso } & \multicolumn{5}{|c|}{$\begin{array}{l}\text { Coincidencias por clase (calificación)/rango } \\
\text { (índice bruto) }\end{array}$} & \multirow[b]{2}{*}{ Coincidencias } & \multirow[b]{2}{*}{ No coinciden } \\
\hline & & 1 & 2 & 3 & 4 & 5 & & \\
\hline NSFa-NSFm & $\mathrm{CH}$ & $0 / 1$ & $1 / 1$ & $19 / 0$ & $0 / 0$ & $0 / 0$ & $20 / 2$ & $7 / 25$ \\
\hline NSFa-ICAUCA & $\mathrm{CH}$ & $0 / 0$ & $4 / 1$ & $13 / 0$ & $0 / 0$ & $0 / 0$ & $17 / 1$ & $10 / 26$ \\
\hline NSFa-CETESB & $\mathrm{CH}$ & $0 / 0$ & $3 / 0$ & $0 / 0$ & $0 / 0$ & $0 / 0$ & $3 / 0$ & $24 / 27$ \\
\hline NSFa-DINIUS & $\mathrm{CH}$ & $0 / 0$ & $0 / 26$ & $0 / 0$ & $0 / 0$ & $0 / 0$ & $0 / 26$ & $27 / 1$ \\
\hline NSFa-IGC & $\mathrm{CH}$ & $0 / 1$ & $4 / 13$ & $0 / 0$ & $0 / 0$ & $0 / 0$ & $4 / 14$ & $23 / 13$ \\
\hline NSFm-ICAUCA & $\mathrm{CH}$ & $0 / 0$ & $1 / 1$ & $9 / 25$ & $0 / 0$ & $0 / 0$ & $10 / 26$ & $17 / 1$ \\
\hline NSFm-CETESB & $\mathrm{CH}$ & $0 / 0$ & $0 / 0$ & $0 / 25$ & $0 / 0$ & $0 / 0$ & $0 / 25$ & $27 / 2$ \\
\hline NSFm-DINIUS & $\mathrm{CH}$ & $0 / 0$ & $0 / 1$ & $1 / 0$ & $4 / 0$ & $0 / 0$ & $5 / 1$ & $22 / 26$ \\
\hline NSFm-IGC & $\mathrm{CH}$ & $0 / 1$ & $1 / 0$ & $10 / 0$ & $0 / 0$ & $0 / 0$ & $11 / 1$ & $16 / 26$ \\
\hline ICAUCA-CETESB & $\mathrm{CH}$ & $0 / 0$ & $13 / 1$ & $0 / 25$ & $0 / 0$ & $0 / 0$ & $13 / 26$ & $14 / 1$ \\
\hline ICAUCA-DINIUS & $\mathrm{CH}$ & $0 / 0$ & $0 / 2$ & $0 / 0$ & $0 / 0$ & $0 / 0$ & $0 / 2$ & $27 / 25$ \\
\hline ICAUCA-IGC & $\mathrm{CH}$ & $0 / 0$ & $12 / 0$ & $11 / 0$ & $0 / 0$ & $0 / 0$ & $23 / 0$ & $4 / 27$ \\
\hline CETESB-DINIUS & $\mathrm{CH}$ & $0 / 0$ & $0 / 1$ & $0 / 0$ & $0 / 0$ & $0 / 0$ & $0 / 1$ & $27 / 26$ \\
\hline CETESB-IGC & $\mathrm{CH}$ & $0 / 0$ & $13 / 0$ & $0 / 0$ & $0 / 0$ & $0 / 0$ & $13 / 0$ & $14 / 27$ \\
\hline DINIUS-IGC & $\mathrm{CH}$ & $0 / 0$ & $0 / 13$ & $0 / 0$ & $0 / 0$ & $0 / 0$ & $0 / 13$ & $27 / 14$ \\
\hline DINIUS - IGC & Agr. & $0 / 0$ & $0 / 0$ & $22 / 0$ & $0 / 0$ & $0 / 0$ & $22 / 0$ & $5 / 27$ \\
\hline DINIUS - IGC & Rec. & $0 / 0$ & $0 / 0$ & $0 / 0$ & $0 / 0$ & $0 / 0$ & $0 / 0$ & $27 / 27$ \\
\hline
\end{tabular}

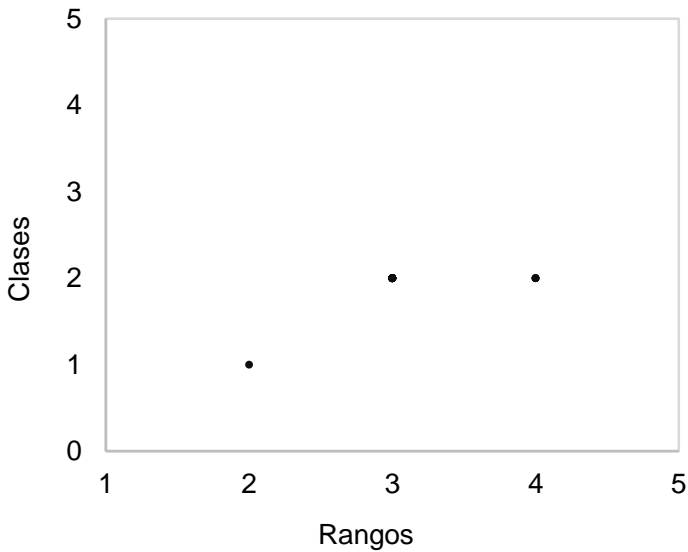

a) NSFm-CETESB por Clases (calificaciones)

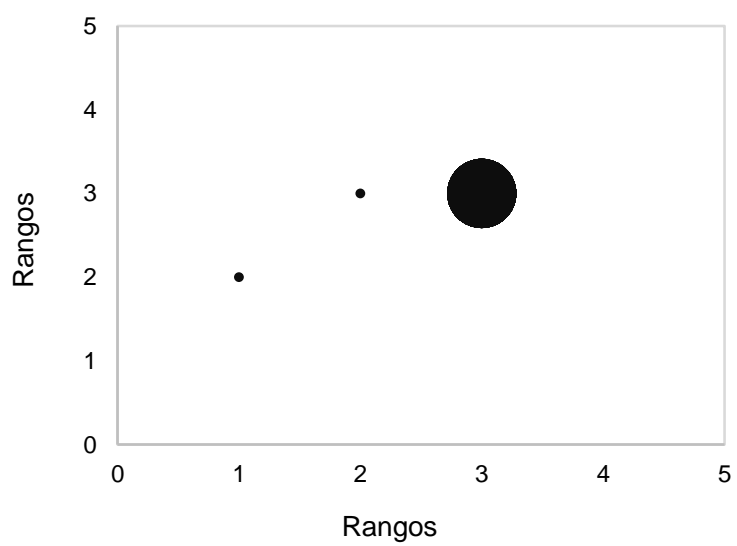

b) NSFm-CETESB por Rangos (índices brutos)

Fig. 2: Comparación de coincidencias entre los índices NSFm-CETESB medidas por clases y rangos en el Rio Ranchería (La Guajira- Colombia), febrero de 2012.

Se observó que un mismo valor del índice bruto puede representar una clase diferente; por ejemplo, un valor de 55 para Dinius indica una clase 4 que corresponde a "mala" en la escala unificada o contaminada tomando la clase original, mientras que para ICAUCA y CETEBB corresponde a una calidad "buena". Como se puede observar en la Tabla 2, para el consumo humano, las coincidencias entre los índices por clases son muy diferentes e inferiores a las de rangos brutos. Lo anterior indica que hay un problema en la escala usada por cada índice para clasificar la calidad del agua. Las diferencias entre los ICAs se deben principalmente al número y/o parámetros incluidos, a los pesos asignados y la función de valor adoptada para determinar los subíndices de calidad. 


\section{Uso agrícola}

De los ICA seleccionados solo Dinius e IGC consideran este uso. Al comparar estos índices se encontró coincidencia en 22 estaciones con clase 3-aceptable, lo que parece indicar que las consideraciones entre estos dos índices son similares. Estos ICAs solo difieren en 5 estaciones donde el IGC muestra una clase 5muy mala y el Dinius clase 2-buena. Para la clase 3, Dinius establece que no requiere tratamiento para la mayoría de los cultivos y el IGC afirma que el agua no es recomendable para cultivos de consumo en crudo, es decir, sí se permite para los demás tipos de cultivos. Es importante anotar que el parámetro más crítico para este uso es CF, el cual hace que se restringa a cierto tipo de cultivos, especialmente los de tallo corto que se consumen en crudo. Similares resultados fueron encontrados en el río Ghezel Ozan-Iran donde la calidad del agua varió desde la clase 3-aceptable a 5-muy mala que restringen el uso agricultura a cierto tipo de cultivos en aquellos lugares donde la presencia de patógeno supera los límites permitidos (Misaghi et al., 2017). Este índice presenta dos inconsistencias: (i) por un lado tomó la clasificación del NSF mientras que para seleccionar los parámetros y criterios de calidad consideraron la guía para uso agrícola de la FAO, no siendo el NSF diseñado para este uso, (ii) los CF terminan definiendo la calidad, pero no fueron considerados en el ICA; evidentemente los resultados habrían sido distintos al incluirlos.

\section{Uso recreativo}

Para este uso no hay coincidencia entre Dinius e IGC. Para Dinius predomina la clase 2-buena indicando una restricción para los deportes de inmersión y precaución si el agua es ingerida por la posibilidad de contener bacterias; en el IGC se encuentra la clase 4-mala, que indica no recomendable su uso directo -esto debido al riesgo para la salud- por tanto requiere un tratamiento y preparación previa, y la clase 5-muy mala, que es inaceptable. A pesar de la diferencia numérica de los índices, en realidad el significado y recomendaciones de clase 2 de Dinius son muy parecidos a los de las clases 4 y 5 del IGC; la razón es que los dos índices se refieren a usos diferentes: el primero trata de uso recreativo en general, en cambio el segundo se refiere a contacto directo. En definitiva, en gran parte de su recorrido el río Ranchería no es apto para el uso recreativo de contacto directo, a pesar que se usa con estos fines en varios sitios. Resultados similares al ICA Dinius fueron encontrados en el río Damour-Líbano donde la calidad fue clase 2-buena a pesar de afirmar que los niveles de estos organismos fueron muy altos, lo que puede indicar riesgos potenciales para la salud de los nadadores (Massoud, 2012). Este índice tiene las mismas inconsistencia del estudio de Misaghi et al. (2017), con la única diferencia que la selección de los parámetros y criterios se hizo tomando varias normativas que regulan el uso recreativo.

Los ICAs se han usado en muchas fuentes hídricas. En aguas subterráneas de Tamil Nadu-India se compararon los resultados del NSFa y NSFm, encontrando diferencias entre ellos, argumentando problemas de eclipsamiento en el NFSa (Ponsadailakshmi et al., 2018). Sin embargo, se recuerda que los valores del NSFm son estructuralmente inferiores, pero la calificación se mide de la misma forma; esto muestra la inconsistencia entre el proceso de agregación de los subíndices y la ponderación de cada parámetro, es decir la determinación de la ecuación del índice, cuyos resultados deben ser consistente con el rango de clase establecido de acuerdo a los valores esperados. A este paso no se le asigna la debida importancia y, si revisamos a Rangeti et al. (2015) y Sutadian et al. (2016), la clasificación no es considerada como parte del proceso en el diseño de un ICA.

Muchas han sido las modificaciones y cambios en la ecuación del ICA de la NSF donde los subíndices de calidad han sido normalizados (normas de calidad para agua potable de la Organización Mundial de la SaludOMS), los pesos reformulados, y hasta hay diferencias en el número de parámetros considerados (Hameed et al., 2010; Sharma et al., 2014; Hussein et al., 2017). Esto genera diferencias entre los ICA al usarlos en la misma fuente hídrica. Exigir a una fuente hídrica los requerimientos establecidos por la OMS dista de la realidad y contexto ya que los ríos o lagos tienen contaminantes antrópicos y naturales propios de su dinámica y por tanto requieren ser tratados para cumplir las exigencias para su consumo. Además de lo anterior estos investigadores consideraron niveles ideales de oxígeno disuelto de 14,6 mg/L lo cual evidentemente es una exigencia adicional extrema, este valor solo es posible a temperatura de $0^{\circ} \mathrm{C}$, la cual difiere sustancialmente de las condiciones climáticas donde se realizaron estos estudios. Es importante agregar que determinar el uso potable a través de un ICA es de cuidado, se puede encontrar buena o excelente calidad incumpliendo los requerimientos de uno o dos parámetros exigidos por la norma, lo que la declararía no aptas para el consumo.

Un paso importante en el diseño de un índice es la selección de sus parámetros o variables (Rangeti et al., 2015; Sutadian et al., 2016; Hou et al., 2016 Sun et al., 2016). Sin embargos, muchas veces no se hace de manera objetiva como en el caso de estas investigaciones citadas aquí que no han considerado los CF como parámetro del índice, lo que es inaceptable desde todo punto de vista, especialmente cuando se trata de evaluar la calidad del agua para consumo humano. Este error puede deberse por considerar los criterios 
establecidos para aguas subterráneas (Sahu y Sikdar, 2008; Ponsadailakshmi, et al., 2018), donde no incuyeron los CF, lo que se justifica por la baja probabilidad de presencia de patógenos (a menos que el acuífero o los pozos se hayan contaminado). Hussein et al. (2017) determinaron calidad buena (clase 2) para beber al excluir la turbidez, y no apta (clase 5) al incluirla. La turbidez (río Al-Gharra-Iraq) alcanzó valores superiores a 1000 UNT y al tener como referente un valor de 0,0 y un estándar de 5 UNT, este parámetro decide sobre la calidad del agua, lo que evidencia un problema en el diseño del ICA. Algo relevante se observa en un estudio realizado en 4 ríos de Italia al comparar 2 índices diseñados de acuerdo con la normativa de la UE: nivel de contaminación de los macro-descriptores (LIM), y a partir del 2010 reemplazado por el Nivel de contaminación macro-descriptores para el estado ecológico (LIMeco). El LIMeco indicó una mejor calidad del agua, lo que se debe en gran medida por la exclusión de los parámetros BOD, DQO y Escherichia coli en este índice (Valeriani et al., 2015).

Para juzgar qué tan correlacionados están los ICAs, se presentan en la Tabla 4 los resultados del coeficiente de correlación de Spearman $(\rho)$ acompañado del nivel de significancia $(p)$ por clases (clasificación) y rangos (índice bruto). La notación $\mathrm{x} / \mathrm{p}$ significa: $\mathrm{x}$ : valor índice de Spearman; $\mathrm{p}$ : nivel de significancia estadística (significativo cuando es $<0,05$ ); NA significa que no aplica porque todos los datos de una serie o de la pareja son iguales. Los valores de $\rho$ indican que las correlaciones por rango son mayores que las obtenidas por la clasificación e incluso todas son significativas, excepto para el IGC.

Tabla 4: Coeficiente de correlación de Spearman para cada pareja de índices por clases y rangos comparados en el río Ranchería (La Guajira-Colombia), febrero de 2012.

\begin{tabular}{|c|c|c|c|c|c|c|c|c|}
\hline \multirow{2}{*}{ Índice } & & NSFm & ICAUCA & CETESB & Dinius & & IGC & \\
\hline & Uso & $\mathrm{CH}$ & $\mathrm{CH}$ & $\mathrm{CH}$ & $\mathrm{CH}$ & $\mathrm{CH}$ & Agr. & Rec. \\
\hline \multicolumn{9}{|c|}{ Valores por clase (calificación) } \\
\hline NSFa & $\mathrm{CH}$ & $0,347 / 0,076$ & $0,402 / 0,038$ & $0,470 / 0,013$ & $0,678 / 0,000$ & $0,402 / 0,038$ & & \\
\hline NSFm & $\mathrm{CH}$ & & $0,458 / 0,016$ & $0,485 / 0,010$ & $0,390 / 0,044$ & $0,275 / 0,166$ & & \\
\hline ICAUCA & $\mathrm{CH}$ & & & $0,189 / 0,345$ & $0,273 / 0,169$ & $0,703 / 0,000$ & & \\
\hline CETESB & $\mathrm{CH}$ & & & & $0,693 / 0,000$ & $0,189 / 0,345$ & & \\
\hline \multirow{3}{*}{ DINIUS } & $\mathrm{CH}$ & & & & & $0,273 / 0,169$ & & \\
\hline & Ag. & & & & & & $0,100 / 0,620$ & \\
\hline & $\operatorname{Rec}$ & & & & & & & $0,400 / 0,039$ \\
\hline \multicolumn{9}{|c|}{ Valores por rango (índices brutos) } \\
\hline NSFa & $\mathrm{CH}$ & $0,721 / 0,000$ & $0,693 / 0,000$ & $0,530 / 0,004$ & NA & $0,189 / 0,345$ & & \\
\hline NSFm & $\mathrm{CH}$ & & $0,999 / 0,000$ & $0,425 / 0,027$ & NA & $0,272 / 0,169$ & & \\
\hline ICAUCA & $\mathrm{CH}$ & & & $0,412 / 0,033$ & NA & $0,273 / 0,169$ & & \\
\hline CETESB & $\mathrm{CH}$ & & & & NA & $0,200 / 0,316$ & & \\
\hline \multirow{3}{*}{ DINIUS } & $\mathrm{CH}$ & & & & & NA & & \\
\hline & Agr. & & & & & & NA & \\
\hline & Rec. & & & & & & & NA \\
\hline
\end{tabular}

En la Tabla 5 se pueden observar los valores de la RMSE que indican una correspondencia mayor cuanto menor es su valor. La notación x/y significa: x: valor para la evaluación en clases (calificación), y: análogo para la evaluación por rangos (índice bruto). Se constata que las mayores coincidencias mostradas en la Tabla 3 tienen los menores valores de RMSE como es ICAUCA-IGC, Dinius-IGC por clase y NSFm-ICAUCA, ICAUCACETESB por rango.

Tabla 5: Raíz del error cuadrático medio (RMSE) para cada pareja de índices por clase de calidad y por rango de valor del índice bruto comparados en el río Ranchería (La Guajira-Colombia), febrero de 2012.

\begin{tabular}{|c|l|c|c|c|c|c|c|c|}
\hline Índice & \multirow{2}{*}{ Uso } & NSFm & ICAUCA & CETESB & DINIUS & \multicolumn{2}{|c|}{ IGC } \\
\cline { 4 - 9 } & $\mathrm{CH}$ & $\mathrm{CH}$ & $\mathrm{CH}$ & $\mathrm{CH}$ & $\mathrm{CH}$ & Agr. & Rec. \\
\hline NSFa & C.H & $0,509 / 0,962$ & $0,609 / 0,981$ & $0,943 / 1,000$ & $1,106 / 0,192$ & $0,609 / 0,756$ & & \\
\hline NSFm & C.H & & $0,793 / 0,192$ & $1,202 / 0,272$ & $0,903 / 0,981$ & $0,839 / 1,569$ & & \\
\hline ICAUCA & C.H & & & $0,720 / 0,192$ & $1,528 / 0,962$ & $0,385 / 1,584$ & & \\
\hline CETESB & C.H & & & & $1,972 / 0,981$ & $0,720 / 1,621$ & & \\
\hline \multirow{3}{*}{ DINIUS } & C.H & & & & & $1,528 / 0,786$ & & \\
\cline { 2 - 8 } & Agr. & & & & & & $0,720 / 1,155$ & \\
\cline { 2 - 8 } & Rec. & & & & & & & $2,776 / 1,732$ \\
\hline
\end{tabular}


Los estudios consultados y citados en este trabajo han reportado la calidad del agua de acuerdo a las diferentes clases obtenida (calificaciones), pero no reportan una comparación análoga a la aquí presentada; y tampoco se ha propuesto unificarlos. Esta investigación enfrenta el problema, de asegurar que los índices lleguen a brindar un juicio, en términos de clase de calidad, realmente apropiado para el particular problema y caso considerado, y de evitar que índices distintos que apuntan a lo mismo den resultados incoherentes. El problema esencial es cómo traducir el valor numérico de un índice en un juicio de calidad que sea realmente entendible y coherente. Este es un problema general de los índices ambientales (no sólo los de calidad del agua), expuesto desde inicios del 2000 (Nardini, 2003), para la generación de información realmente útil para la toma de decisiones en problemas multiobjetivo, multiactores caracterizados por presencia de conflictos de intereses. En este sentido, se reconoce que hay un paso en la construcción del índice que inevitablemente es subjetivo; este hecho no debe ser razón para rechazarlos, sino más bien para fortalecer su proceso de construcción.

En la práctica común, los índices de calidad del agua no miden directamente los atributos que describen el problema de interés; el resultado final -es decir el valor numérico del índice- es por tanto fruto típicamente de la asignación de juicios subjetivos que, implícitamente, cubren dos funciones bien diferentes: i) sustituir un modelo causa-efecto que relacione los atributos realmente de interés (y no explicitados) a los parámetros de calidad del agua considerados (causas); ii) agregar los distintos aspectos (atributos) en un juicio final holístico que por definición no puede ser subjetivo. Mientras la primera función tendría que ser objetiva, la segunda inevitablemente es subjetiva, lo que se conoce como elicitation en la literatura anglosajona. El hecho de no separar y explicitar estas dos funciones genera inevitablemente las incoherencias observadas, es decir que ICAs diferentes que apuntan al mismo objetivo y deberían producir juicios sustancialmente equivalentes o similares sean en realidad bien distante. Se trata de un verdadero problema. Análogamente, nada asegura que el mismo índice produzca juicios coherentes cuando se aplique a regiones ecológicas o tipologías fluviales diferentes, incluso al interior de un mismo país. De acuerdo a lo anterior, se debe hacer un esfuerzo para construir índices basados en atributos naturales, separar y explicitar el modelo de relación causa efecto entre los indicadores de calidad del agua, es decir los parámetros de calidad medibles y los atributos naturales, tarea por realizar -de acuerdo con Sutadian et al. (2016)- a través de unos paneles de discusión con fuerte participación de los grupos sociales interesados.

Los modelos causa-efecto, dependen de los atributos ("efectos" y "causas") considerados esenciales para describir la calidad del agua y la satisfacción objeto de interés (ej. idoneidad para uso recreativo de contacto) y representan los mecanismos/procesos que ligan los dos. Este modelo puede ser mecanicista (modelo basado en leyes físicas o su abstracción conceptual), empírico (basado en -un gran número deobservaciones) u holístico (basado típicamente en paneles interdisciplinarios de expertos como ecólogo, epidemiólogo, ingenieros ambientales, entre otros). En cualquiera de estos casos, el modelo típicamente podría igualmente tener validez general al menos en un ámbito fisiográfico definido (eco-región) y para una dada tipología de ríos.

El punto clave abordado en este artículo es conceptualmente sucesivo al apenas discutido y es cómo asegurar que la diferenciación en términos de calificación, es decir la clase, determinada por un cierto índice sea la misma establecida por otros índices que apuntan al mismo objetivo. Esta exigencia no es para nada nueva y de hecho tiene un importante antecedente en la UE. La UE definió a través de su Directiva 2000/60/CE un marco comunitario de actuación en el ámbito de la política de aguas. El insumo base ha sido la clasificación de la calidad de los cuerpos hídricos con la exigencia de asegurar la coherencia entre los distintos enfoques de evaluación en los países miembros. Para ello, la UE impulsó y llevó a cabo un complejo y largo ejercicio de intercalibración conformando para ello una red de grupos/puntos de control en cada estado y en cada región ecológica (Comisión Europea, 2013). El criterio adoptado por la UE es dotarse de una escala que mide la distancia del estado de referencia: cuanto más cercano, mejor es la calidad ecológica. Para ello, se ha tenido que resolver con anterioridad dos problemas difíciles: (i) distinguir tipologías de cuerpos hídricos y (ii) definir el estado de referencia de cada río, es decir las condiciones que tendrían sin perturbación antrópica. Para la definición de tipologías se han adoptado varios métodos, siendo el principal una clasificación basada en características naturales del río tales como: tamaño de la cuenca, altitud media, geología entre otras.

\section{DISCUSIÓN FINAL}

Las mayores coincidencias, mejor correlación y menores desviaciones fueron obtenidas para los índices brutos (en parejas de índices diferentes a las que mostraron coincidencias por clase). Esto evidencia la carencia del proceso de traducción de los valores de los índices a juicios ambientales (calificación), por lo que se puede afirmar que en los índices se obtienen juicios diferentes y por tanto hay que usarlos con mucha precaución al momento de planificar el uso del agua del río Ranchería. A pesar de lo anterior se pude decir que las clases predominantes en el río Ranchería para el uso de consumo humano fueron: 2-buena, 3aceptable y 4-mala. Para uso agrícola predominó clase 3-aceptable y para la recreación no hubo coincidencia 
de clase pero la recomendación es no usarse para contacto directo sin tratamiento previo. Los CF, oxígeno disuelto y turbidez son los parámetros que más afectan, derivado de los procesos antrópicos desarrollados en la cuenca como ha sucedido en otras investigaciones (Massoud, 2012; Sun et al., 2016; Misaghi et al., 2017). Estos resultados fueron entregados a entidad competente para que planifique de manera más adecuada los usos posibles del río Ranchería.

El problema central de las inconsistencias de los ICAs es el carácter fuertemente subjetivo que existe al definir la función de valor que expresa el equivalente entre la variable y su nivel de calidad a través de una función específica y la manera en que se agregan distintos atributos. Estas funciones no son universales, por tanto no se puede aplicar en todas las áreas geográficas (Tyagi et al., 2013). De los índices usados en el presente trabajo el único que no usa una verdadera función de valor es el IGC. Por otro lado, la técnica usada por Horton (comité de expertos) y por el Dinius y con el método Delphi (cuestionario) para el NSF -en ambos casos para decidir si incluir o no una variable- es fuertemente criticado por incorporar opiniones subjetivas: los expertos con frecuencia no estaban de acuerdo con la importancia relativa de las variables, juicio que podría ser cuestionado por otro panel de expertos; además, los juicios de los expertos estaban sesgados por sus antecedentes profesionales (Rangeti et al., 2015). La razón a la raíz de este problema es en realidad la indicada por Nardini (2003) ya recordada, es decir el incorporar implícitamente dentro el mismo índice un modelo causa-efecto. Del NSF se han derivado muchos índices, entre ellos CETESB, Dinius e ICAUCA que casi en su totalidad siguen la misma técnica de agregación de la NSF sea aditiva o multiplicativa.

En general los ICAs se modifican de acuerdo a los criterios normativos a respetar, pero su estructura adopta una función de agregación análoga (en su mayoría parecida a la del NSF), mientras que los pesos han sido modificados a criterio de los investigadores. De acuerdo con Rangeti et al. (2015) y Sutadian et al. (2016) esto denota mucha subjetividad e incertidumbre involucradas en los pasos para desarrollar y aplicar estos índices. Algunos han utilizado técnicas como el coeficiente de correlación de Pearson, análisis de componentes principales, análisis de conglomerados y el análisis discriminante para la selección de los parámetros y reducir la colinealidad e incertidumbre (Hou et al., 2016; Sun et al., 2016); pero estas técnicas siguen siendo poco comunes posiblemente debido a sus complicados procedimientos estadísticos y por tanto muchos estudios continúan utilizando la opinión de expertos junto con los hallazgos de la literatura y criterios de los investigadores (Rangeti et al., 2015).

Sutadian et al. (2016) notan además que los métodos estadísticos siguen siendo subjetivos ya que se basan en los datos proporcionados para el análisis, sería más acertado entonces seleccionar los parámetros con un análisis causa-efecto (que debe y puede ser objetivo), pero para ello es necesario tener una medida de la satisfacción de acuerdo al uso (ej. morbilidad). Para reducir la subjetividad en la asignación de los pesos, Sutadian et al. (2017) recomiendan adoptar un proceso de jerarquía analítica ideado por Satty en 1980. Este método sin embargo recopila los juicios basándose en pensamientos, experiencias y conocimiento de los participantes, por tanto igual a los demás métodos existe subjetividad, por otro lado se puede aplicar con diferentes escalas de puntuación por lo que se pueden obtener pesos distintos. Todas estas metodologías y procedimientos para el diseño de índices pueden ser válidos, pero el paso usado para determinar su calificación es en general muy débil y genera las incoherencias antes discutidas. Esto se puede solucionar a través de unos paneles de discusión con fuerte participación de los grupos sociales interesados.

\section{CONCLUSIONES}

De acuerdo al trabajo presentado y a los resultados obtenidos, se pueden plantear las siguientes conclusiones principales:

1.- Al comparar los índices se presentaron importantes diferencias entre ellos, y mucho más en términos de las clases (calificación), por tanto queda la incertidumbre acerca del uso de los ICAs para fines de planificación y manejo del recurso hídrico. Esta dificultad no solo afecta al río Ranchería, por el contrario es a nivel mundial.

2.- Para el diseño de los índices es necesario enfrentar las incoherencias empezando a separar el modelo causa-efecto del juicio holístico de calidad, o por lo menos, llegar a "calibrar" los índices de manera que sean más coherentes, refinando los juicios subjetivos y articulándolos por eco-regiones para que sean más representativos. En el proceso de construcción es oportuno considerar el análisis de incertidumbre y sensibilidad.

3.- El agua del río Ranchería no debe usarse para contacto directo sin previo tratamiento y antes de su consumo humano requiere de procesos de potabilización especialmente desinfección rigurosa. Lo anterior por las altas concentraciones de organismos coliformes que favorece la presencia de enfermedades en la salud humana. 
4.- Todas las medidas, acciones y recomendaciones para optimizar los usos del río Ranchería fueron informadas a las autoridades competentes a través del PORH de este río. Estos resultados son de utilidad para planificar y optimizar el uso de esta fuente, pero deben usarse con precaución por las incoherencias encontradas en los ICAs utilizados.

\section{AGRADECIMIENTOS}

Se agradece a Corpoguajira por la disponibilidad de datos históricos; a la Universidad de La Guajira, y al equipo de trabajo del proyecto PORH en especial a Mayiber Rangel y Daliana Pinto por el procesamiento de datos; a Lislie Zúñiga y Wendy Ávila por sus aportes en la concepción y aplicación del índice IGC, y Yesenia Zuñiga por la edición de tablas, figuras y organización del documento.

\section{REFERENCIAS}

APHA-American Public Health Association; AWWA-American Water Works Association y WEF-Water Environment Federation, Standard methods for the examination of water and wastewater, $21^{\text {st }}$ edition, 1-1368. Ed. American, Washington D.C, USA (2005).

Blum, D. y Holling, H., Spearman's law of diminishing returns. $A$ meta-analysis, doi: https://doi.org/10.1016/j.intell.2017.07.004. Intelligence, 65, 60-66 (2017).

Brierley, G., Fryirs, K., Cook, N., Outhet, D., Raine, A., Parsons, L., y Healey, M., Geomorphology in action: Linking policy with on-the-ground actions through applications of the River Styles framework, doi: http://doi.org/10.1016/j.apgeog.2011.03.002, Applied Geography. 31(3), 1132-1143 (2011).

CETESB - Companhia De Tecnologia De Saneamento Ambiental, Índices de qualidade das águas interiores do Estado de São Paulo, $21^{\text {st }}$ edición, 1-537. Visiva Design Gráfico, São Paulo, Brasil (2006).

Comisión Europea, Directiva 2013/480/UE, Los valores de las clasificaciones de los sistemas de seguimiento de los Estados miembros a raíz del ejercicio de intercalibración, (en la web: www.boe.es/doue/2013/266/L00001-00047.pdf, acceso: 21 de Febrero 2016) Diario Oficial de las Comunidades Europeas, Bruselas, Bélgica (2013).

Corpoguajira-Corporación Autónoma Regional De La Guajira, Plan de ordenamiento de recursos hídricos (PORH) de los ríos Ranchería, Tomarrazón, Carraipía, La Guajira- Colombia, (en la web: http://corpoguajira.gov.co/wp/?s=rancher\%C3\%ADa, acceso: 20 de octubre 2015) (2012).

Corpoguajira-Corporación Autónoma Regional De La Guajira y Ricerca e Cooperazione, Plan de ordenación y manejo de la cuenca de Camarones Tomarrazón (POMCA), Proyecto apoyado por la ONG internacional, Capitulo calidad del agua superficial y subterránea, (en la web: http://corpoguajira.gov.co/wp/pomca-tomarrazon-camarones, acceso: 20 de octubre 2015) (2007).

CVC- Corporación Autónoma Regional Del Valle Del Cauca, Estudio de la calidad del agua del río Cauca y sus principales tributarios mediante la aplicación de índices de calidad y contaminación. Reporte del proyecto 0168, octubre de 2004, (en http://www.cvc.gov.co/cvc/Mosaic/dpdf2/Volumen10/1-ECARCpag1-158.pdf, acceso: 20 de marzo de 2015) (2004).

Dinius, S.H., Design of an index of water quality, doi: 10.1111 / j.1752-1688.1987.tb02959.x, JAWRA Revista de la Asociación Americana de Recursos de Agua, 23 (5), 833-843 (1987).

Hameed, A., Alobaidy, M. J., Abid, H. S., y Maulood, B. K. Application of Water Quality Index for Assessment of Dokan Lake Ecosystem, Kurdistan Region, Iraq, doi: http://doi.org/10.4236/jwarp.2010.29093, Journal of Water Resource and Protection, 2(9), 792-798 (2010).

Hou, W., Sun, S., Wang, M., Li, X., Zhang, N., Xin, X., Jia, R., Assessing water quality of five typical reservoirs in lower reaches of Yellow River, China: Using a water quality index method, doi: . http://doi.org/10.1016/j.ecolind.2015.09.030, Ecological Indicators, 61, 309-316 (2016).

Hurley, T., Sadiq, R., y Mazumder, A., Adaptation and evaluation of the Canadian Council of Ministers of the Environment Water Quality Index (CCME WQI) for use as an effective tool to characterize drinking source water quality, doi:10.1016 / j.watres.2012.03.061, Water Research, 46(11), 3544-3552 (2012).

Hussein E.S., y Abed, S.A., Water quality index for Al-Gharraf River, southern Iraq, doi: http://doi.org/10.1016/j.ejar.2017.03.001, Egyptian Journal of Aquatic Research, 43(2), 117-122 (2017).

Massoud, M.A., Assessment of water quality along a recreational section of the Damour River in Lebanon using the water quality index, doi: http://doi.org/10.1007/s10661-011-2251-z Environmental Monitoring and Assessment, 184(7), 41514160 (2012).

Medeiros, A.C., Freitas Faial, K.R., Freitas Faial, K.D., da Silva Lopes, I.D., de Oliveira Lima, M., Guimarães, R. M., y Mendonça, N. M., Quality index of the surface water of Amazonian rivers in industrial areas in Pará, Brazil, doi: http://doi.org/10.1016/j.marpolbul.2017.09.002, Marine Pollution Bulletin, 123(1-2), 156-164 (2017).

Mentaschi, L., Besio, G., Cassola, F., y Mazzino, A. Problems in RMSE-based wave model validations, doi: . http://doi.org/10.1016/j.ocemod.2013.08.003, Ocean Modelling, 72, 53-58 (2013). 
Ministerio De Agricultura, Decreto 1594 de 1984, Por el cual se reglamenta los usos del agua y residuos líquidos, (en la web: www.ins.gov.co, acceso 28/01/2016), Bogotá, Colombia (1984)

Misaghi, F., Delgosha, F., Razzaghmanesh, M., y Myers, B., Introducing a water quality index for assessing water for irrigation purposes: A case study of the Ghezel Ozan River, doi: http://doi.org/10.1016/j.scitotenv.2017.02.226, Science of the Total Environment, 589, 107-116. (2017).

Nardini, A., A systematic approach to build evaluation indices for environmental decision making with active public involvement, di Economia delle fonti di Energia e dell'Ambiente, 46 (1-2),189-215 (2003).

Ocampo-Duque, W., Osorio, C., Piamba, C., Schuhmacher, M., y Domingo, J.L., Water quality analysis in rivers with nonparametric probability distributions and fuzzy inference systems: Application to the Cauca River, Colombia, doi: http://doi.org/10.1016/j.envint.2012.11.007, Environment International, 52, 17-28. (2013).

Ponsadailakshmi, S., Sankari, S.G., Prasanna, S.M., y Madhurambal, G., Evaluation of water quality suitability for drinking using drinking water quality index in Nagapattinam district, Tamil Nadu in Southern India, doi: http://doi.org/10.1016/j.gsd.2017.10.005, Groundwater for Sustainable Development, 6, 43-49 (2018).

Rangeti, I., Dzwairo, B., Barratt, G., y Otieno, F. Ecosystem-specific water quality indices, doi: http://doi.org/10.2989/16085914.2015.1054341, African Journal of Aquatic Science, 40(3), 227-234 (2015).

Rivera, N.R., Encina, F., Palma, R., y Mejias, P., La Calidad de las Aguas en el Curso Superior y Medio del Río Traiguén. IX Región-Chile, doi: http://doi.org/10.1612/inf.tecnol.3920it.07, Info. Tecnol. 20(4), 75-84 (2009).

Sahu, P., y Sikdar, P.K. Hydrochemical framework of the aquifer in and around East Kolkata Wetlands, West Bengal, India, doi: http://doi.org/10.1007/s00254-007-1034-x, Enviro.Geol. 55(4), 823-835 (2008).

Sharma, P., Meher, P.K., Kumar, A., Gautam, Y.P., y Mishra, K.P., Changes in water quality index of Ganges river at different locations in Allahabad, doi: http://doi.org/10.1016/j.swaqe.2014.10.002, Sustainability of Water Quality and Ecology, 3-4, 67-76 (2014).

Sun, W., Xia, C., Xu, M., Guo, J., y Sun, G., Application of modified water quality indices as indicators to assess the spatial and temporal trends of water quality in the Dongjiang River, doi: . http://doi.org/10.1016/j.ecolind.2016.01.054, Ecological Indicators, 66, 306-312 (2016).

Sutadian, A.D., Muttil, N., Yilmaz, A.G., y Perera, B.J.C. Development of river water quality indices-a review, doi: http://doi.org/10.1007/s10661-015-5050-0, Environ. Monit. Assess. 188, 58. (2016).

Sutadian, A.D., Muttil, N., Yilmaz, A.G., y Perera, B.J.C., Using the Analytic Hierarchy Process to identify parameter weights for developing a water quality index, doi: http://doi.org/10.1016/j.ecolind.2016.12.043, Ecological Indicators, 75, 220-233 (2017).

Tyagi, S., Sharma, B., Singh, P., y Dobhal, R. Water Quality Assessment in Terms of Water Quality Index, doi: http://doi.org/10.12691/ajwr-1-3-3, American Journal of Water Resources, 1(3), 34-38. (2013).

Valeriani, F., Zinnà, L., Vitali, M., Romano Spica, V., \& Protano, C., River water quality assessment: Comparison between old and new indices in a real scenario from Italy, http://doi.org/10.1080/15715124.2015.1012208, Intl. J. River Basin Management, 13(3), 325-331 (2015). 